\title{
Point-of-care Ultrasound Diagnosis of Type B Aortic Dissection on the Suprasternal Notch View
}

\author{
Richard N. Wang ${ }^{1}$, Michelle Escobar ${ }^{1}$, Shamicka North ${ }^{2}$, Judy Lin ${ }^{1}$ \\ 1. Emergency Medicine, Maimonides Medical Center, Brooklyn, USA 2. Pediatric Emergency Medicine, Maimonides \\ Medical Center, Brooklyn, USA
}

Corresponding author: Richard N. Wang, riwang@maimonidesmed.org

\begin{abstract}
Aortic dissection (AD) is a life-threatening but uncommon and challenging diagnosis to make in the emergency department. Cardiac point-of-care ultrasound (POCUS) is often used to evaluate patients with chest pain and may be used to rapidly diagnose Stanford type AD on the suprasternal notch view if there is the visualization of a dissection flap. In contrast, a diagnosis of type B AD on the suprasternal notch view is rare and has only been reported in one previous case report. We report the case of a patient who presented with chest pain and was accurately diagnosed with type $\mathrm{B} A \mathrm{D}$ using the suprasternal notch view.
\end{abstract}

Categories: Cardiac/Thoracic/Vascular Surgery, Emergency Medicine

Keywords: aortic dissection, emergency ultrasound, point of care ultrasound, suprasternal

\section{Introduction}

Aortic dissection $(\mathrm{AD})$ is a life-threatening but uncommon and challenging diagnosis to make in the emergency department (ED). While a sudden onset of severe pain is the most common presenting complaint, it can have a wide range of manifestations and classic findings are often absent [1]. Computed tomography angiography (CTA) is the most common initial assessment performed and has high sensitivity and specificity [1-2]. The Stanford classification divides AD into type A, which involves the ascending aorta, and type B, which is limited to the aorta distal to the left subclavian artery. Cardiac point-of-care ultrasound (POCUS) is often used to evaluate patients with chest pain and may be used to rapidly diagnose type $\mathrm{A} A \mathrm{D}$ in the suprasternal notch view if there is the visualization of a dissection flap [3]. In contrast, a diagnosis of type B $\mathrm{AD}$ in the suprasternal notch view is rare and has only been reported in one previous case report. We report the case of a patient who presented with chest pain and was accurately diagnosed with type B AD using the suprasternal notch view.

Received 10/10/2019

Review began 10/14/2019 Review ended 10/23/2019 Published 10/27/2019

\section{() Copyright 2019}

Wang et al. This is an open access article distributed under the terms of the Creative Commons Attribution License CC-BY 3.0., which permits unrestricted use, distribution, and reproduction in any medium, provided the original author and source are credited.

\section{Case Presentation}

A 77-year-old male with a history of hypertension presented to the ED with the chief complaint of chest pain. He described excruciating substernal chest pain that was sudden onset, non-radiating, non-pleuritic, and without associated nausea, vomiting, diaphoresis, or shortness of breath.

His initial vitals were as follows: temperature $97.7^{\circ} \mathrm{F}$, heart rate 57 beats/minute, respiratory rate 18 breaths/min, blood pressure $131 / 64 \mathrm{mmHg}$, and oxygen saturation $95 \%$ on room air. His physical exam was unremarkable. His laboratory results were notable for a white blood cell (WBC) count of 15,100 cells per microliter, normal basic metabolic panel, and two sets of cardiac troponin I negative. An electrocardiogram (ECG) showed ST-elevation in V2 and V3 without reciprocal changes (Figure 1). Chest X-ray showed bibasal atelectasis and fullness of the right hilum (Figure 2).

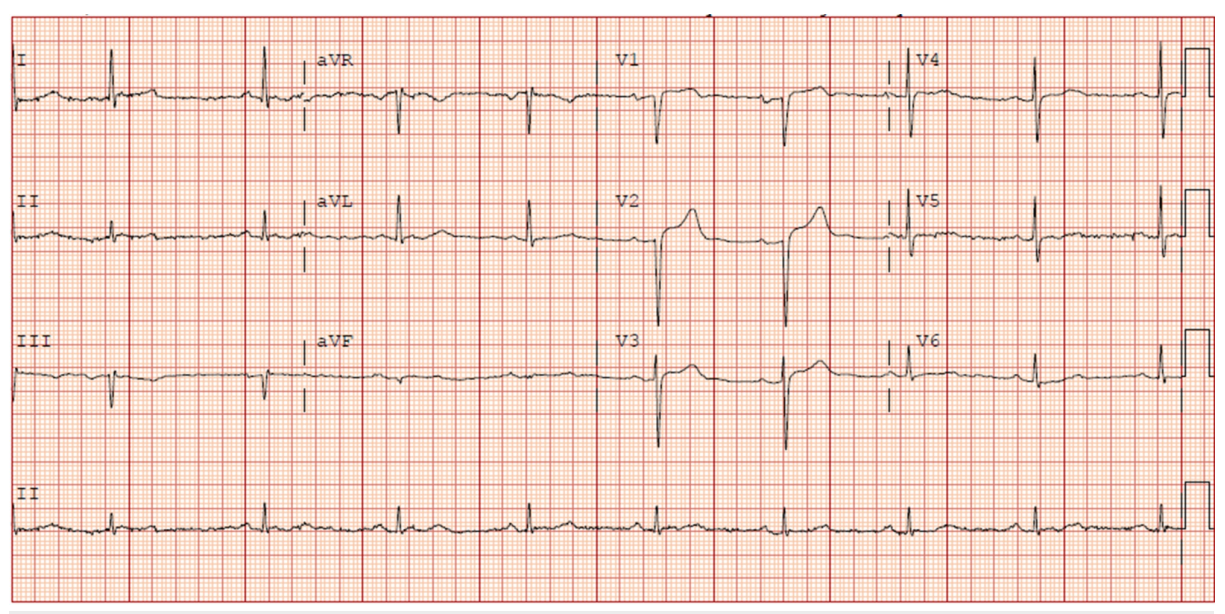




\section{Cureus}

\section{FIGURE 1: ECG}

ECG: electrocardiogram

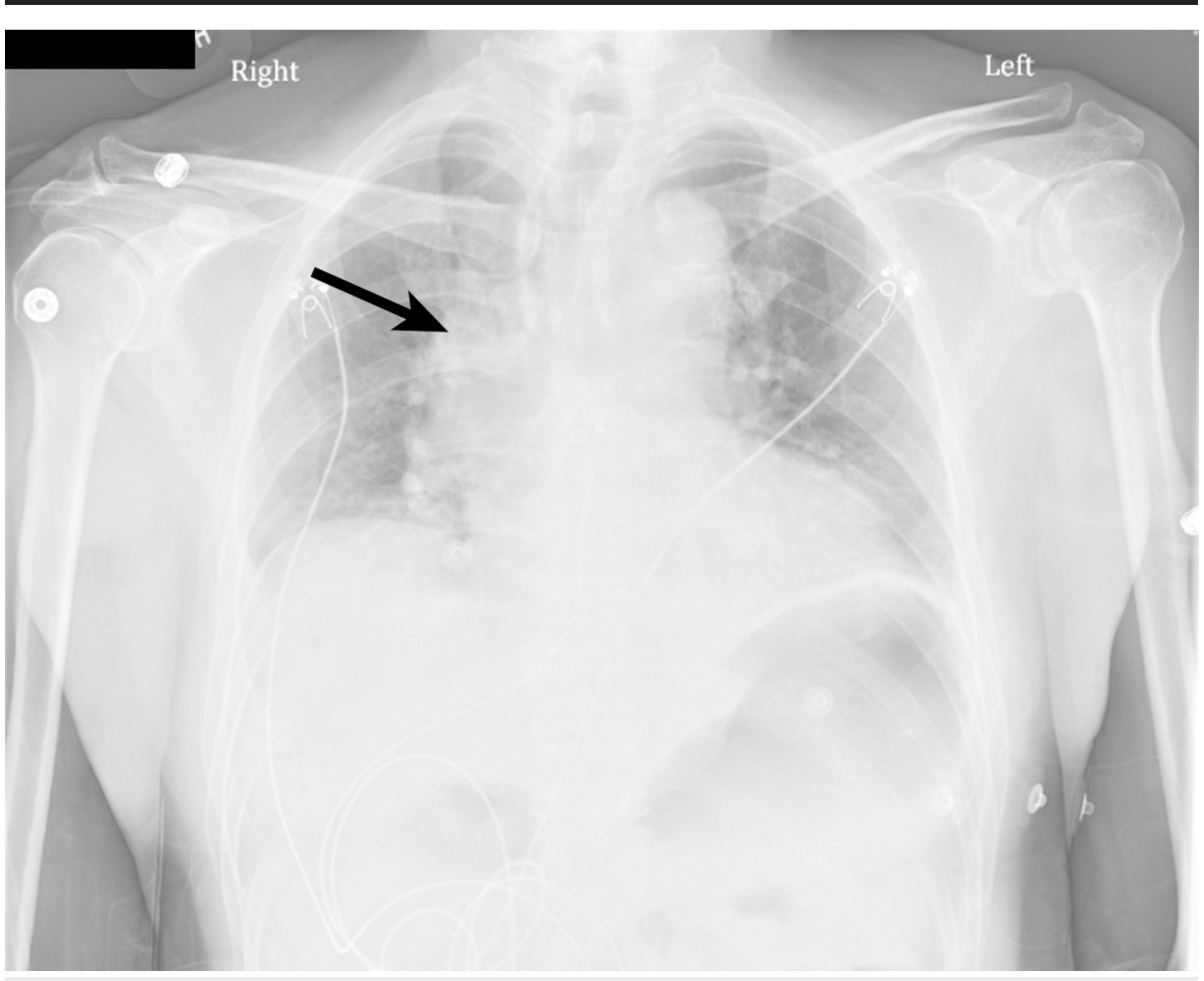

FIGURE 2: CXR with fullness of the right hilum

CXR: chest X-ray

About six hours after initial presentation, the patient was noted to be hypertensive to 200/95 mmHg with concern of pain moving to the middle of the abdomen. POCUS of the abdominal aorta at that time revealed an area concerning for an intimal flap, which was more clearly appreciated on the cardiac suprasternal notch view (Videos 1-3, Figure 3).

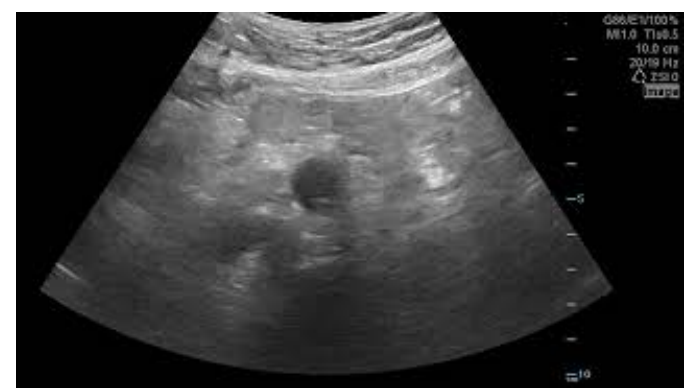

VIDEO 1: Abdominal aorta (transverse view) with suggestion of dissection flap

View video here: https://www.youtube.com/watch?v=mmWiSrEzR5o 


\section{Cureus}

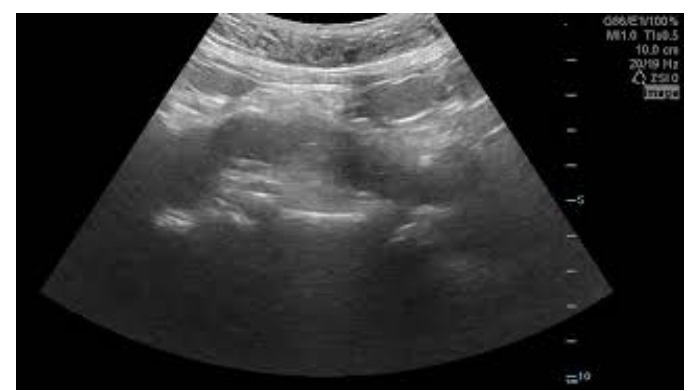

VIDEO 2: Abdominal aorta (longitudinal view)

View video here: https://www.youtube.com/watch?v=zHseDVFPOrE

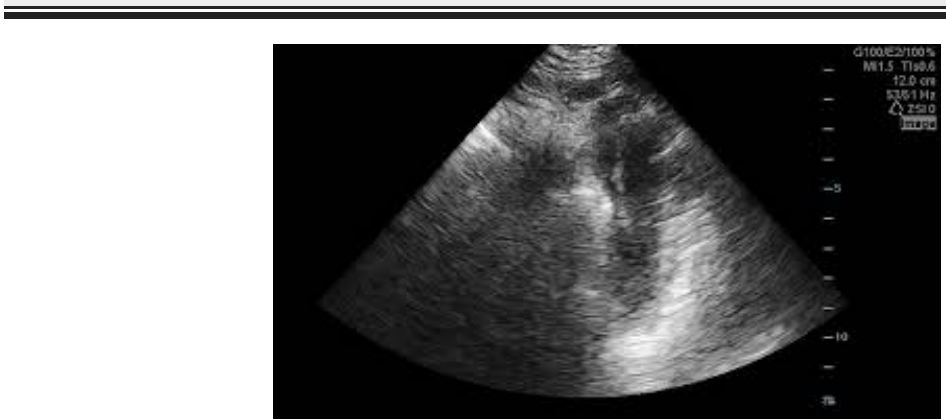

VIDEO 3: Suprasternal notch view with clear visualization of dissection flap

View video here: https://www.youtube.com/watch?v=vvMbrliLyZM

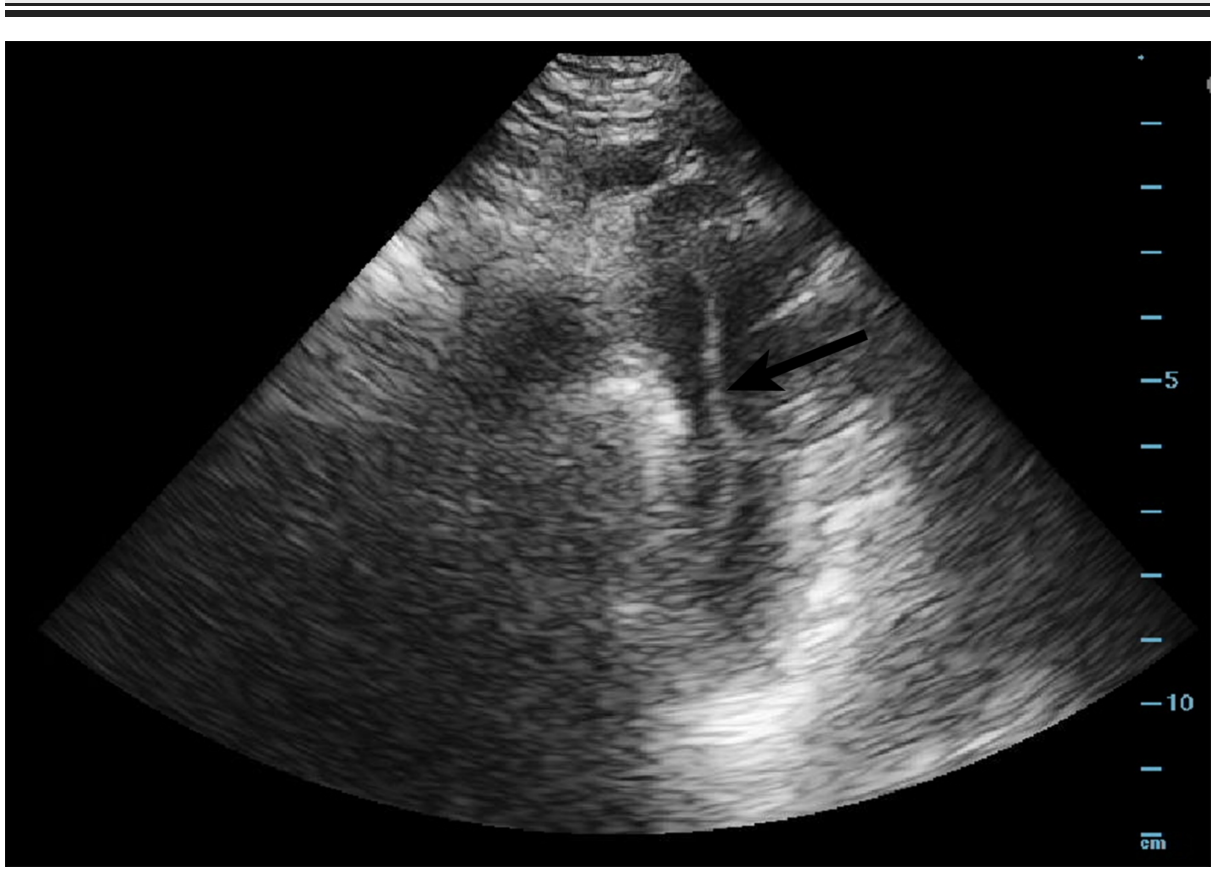

FIGURE 3: Suprasternal notch view with visualization of a dissection flap

A CTA was performed and showed a type B AD with arch involvement beginning just distal to the takeoff of the left subclavian artery and extending to the infrarenal abdominal aorta (Figure 4). 


\section{Cureus}

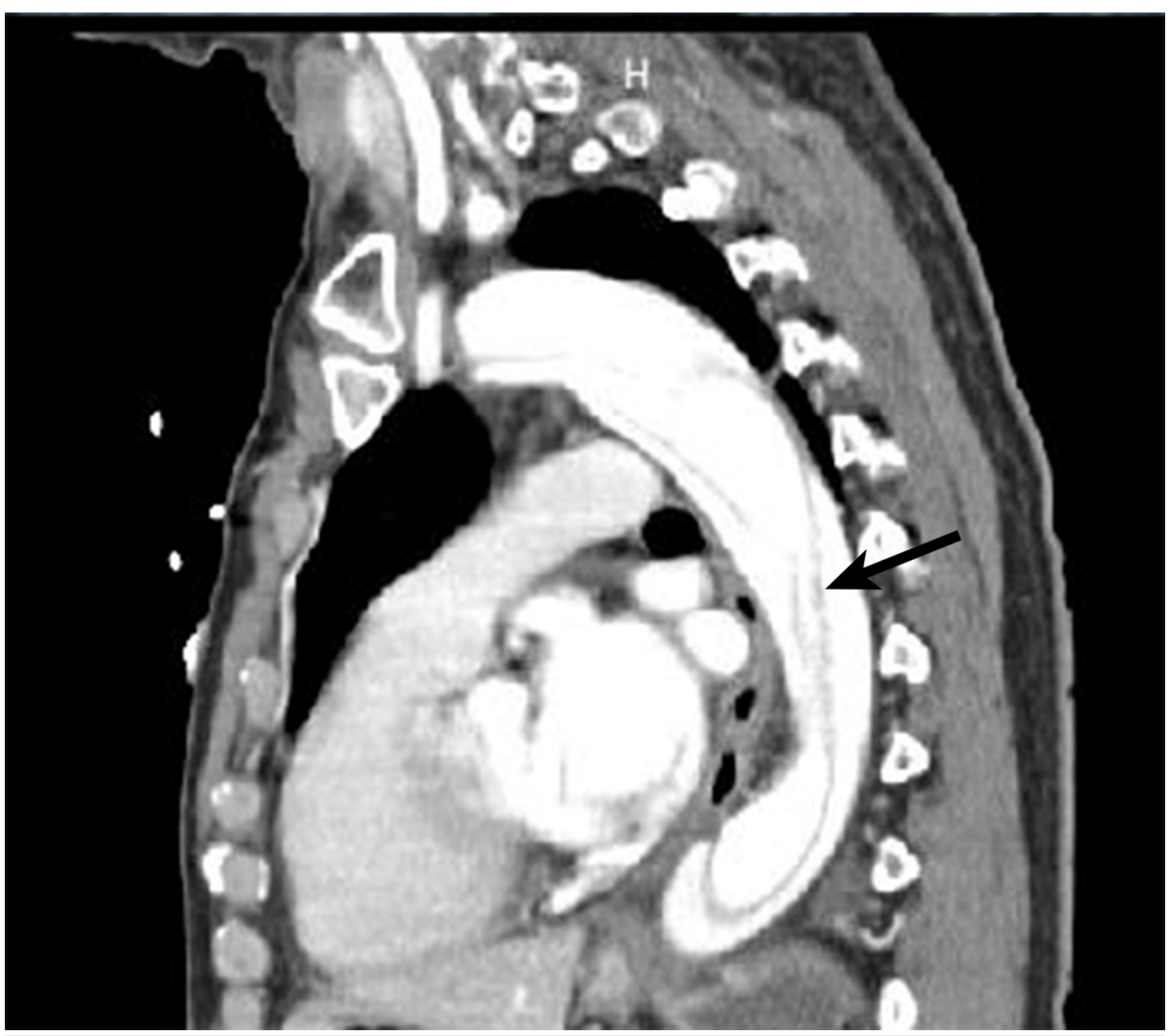

\section{FIGURE 4: CTA showing type B AD with arch involvement}

The patient was started on an esmolol drip and admitted to the cardiothoracic surgery service.

An inpatient transthoracic echocardiogram (TTE) revealed a mild to moderate aneurysm of the ascending aorta. A repeat CTA on hospital day two showed no significant interval change of the AD. The patient was medically managed with anti-hypertensives and discharged on hospital day seven. Follow-up CTAs in three weeks, four months, and one year showed stable dissection.

\section{Discussion}

$\mathrm{AD}$ is an uncommon diagnosis, with an estimated prevalence of 5,000 to 10,000 cases in the United States per year [4]. It is a difficult diagnosis for emergency physicians to make, and $15 \%-43 \%$ of cases are missed on initial presentation [4]. Missed diagnosis of $\mathrm{AD}$ is most common in patients with a working diagnosis of acute coronary syndrome, which is a much more prevalent condition [2]. The Stanford classification divides $\mathrm{AD}$ into type $\mathrm{A}$, which involves the ascending aorta, and type $\mathrm{B}$, which is limited to the aorta distal to the left subclavian artery. Type $\mathrm{A} A \mathrm{AD}$ is quoted to have a $1 \%$ per hour mortality and, even with surgical intervention, has a reported 30-day mortality of $17 \%$ [5]. Type B AD, typically managed medically, has a reported mortality of about $11 \%$, and those on whom surgery is performed have a mortality rate of $31.5 \%$ [1]. CTA, magnetic resonance imaging (MRI), and transesophageal echocardiography (TEE) are all reliable diagnostic imaging modalities, reaching $100 \%$ sensitivity, and with CTA reaching $100 \%$ specificity [2].

Numerous case reports and series have demonstrated that POCUS performed by emergency physicians can diagnose abdominal and thoracic $\mathrm{AD}$ by the visualization of the dissection flap via the traditional transabdominal and transthoracic, specifically the parasternal long axis, views [6-9]. The transthoracic view has the disadvantage of visualizing only the proximal aorta and is thus not able to evaluate for type B AD. The suprasternal notch view, not part of routine cardiac POCUS, can be used to rapidly diagnose AD, primarily for type $\mathrm{A} A \mathrm{AD}$. The view is obtained by placing the cardiac probe $(2-4 \mathrm{MHz})$ over the sternal notch, indicator towards the head of the patient, and then slowly rotating the probe clockwise towards the patient's left shoulder. One reference notes that this view is only useful for visualization of type A AD and not type B [3]. However, our case illustrates a clearly visualized type B AD with this view. One prior case report in the literature diagnosed a type B AD on suprasternal notch view in a similar manner [10]. As the innominate, left common carotid, and left subclavian arteries can be visualized in this view, either type A or B AD can be diagnosed [10].

\section{Conclusions}


Cardiac POCUS can be used to rapidly diagnose $\mathrm{AD}$ if there is visualization of a dissection flap. While more commonly used to evaluate for type $\mathrm{A} A \mathrm{D}$, it is important to know that the suprasternal notch view may also be used to visualize a type $\mathrm{B} A \mathrm{D}$. The suprasternal notch view may provide imaging of the descending portion of the thoracic aorta, which can be missed by the cardiac parasternal long-axis view and the abdominal aorta view. In patients in whom there is suspicion for $\mathrm{AD}$, the suprasternal notch view may be incorporated into a cardiac POCUS, as it may aid in the diagnosis of type B AD not visualized by other cardiac and aorta views.

\section{Additional Information \\ Disclosures}

Human subjects: Consent was obtained by all participants in this study. Conflicts of interest: In compliance with the ICMJE uniform disclosure form, all authors declare the following: Payment/services info: All authors have declared that no financial support was received from any organization for the submitted work. Financial relationships: All authors have declared that they have no financial relationships at present or within the previous three years with any organizations that might have an interest in the submitted work. Other relationships: All authors have declared that there are no other relationships or activities that could appear to have influenced the submitted work.

\section{References}

1. Hagan PG, Nienaber CA, Isselbacher EM, et al.: The International Registry of Acute Aortic Dissection (IRAD): new insights into an old disease. JAMA. 2000, 283:897-903. 10.1001/jama.283.7.897

2. Hiratzka LF, Bakris GL, Beckman JA, et al.: 2010 ACCF/AHA/AATS/ACR/ASA/SCA/SCAI/SIR/STS/SVM guidelines for the diagnosis and management of patients with thoracic aortic disease. A report of the American College of Cardiology Foundation/American Heart Association Task Force on Practice Guidelines, American Association for Thoracic Surgery, American College of Radiology, American Stroke Association, Society of Cardiovascular Anesthesiologists, Society for Cardiovascular Angiography and Interventions, Society of Interventional Radiology, Society of Thoracic Surgeons, and Society for Vascular Medicine. Circulation. 2010, 121:266-369. 10.1161/CIR.0b013e3181d4739e

3. Fernando SM, Kisilewicz M, Millington SJ: View from the top: point-of-care ultrasound diagnosis of type A aortic dissection using the suprasternal view. J Emerg Med. 2018, 54:13-14. 10.1016/j.jemermed.2017.09.019

4. Strayer RJ, Shearer PL, Hermann LK: Screening, evaluation, and early management of acute aortic dissection in the ED. Curr Cardiol Rev. 2012, 8:152-157. 10.2174/157340312801784970

5. Conzelmann LO, Weigang E, Mehlhorn U, et al.: Mortality in patients with acute aortic dissection type A: analysis of pre- and intraoperative risk factors from the German Registry for Acute Aortic Dissection Type A (GERAADA). Eur J Cardiothorac Surg. 2016, 49:44-52. 10.1093/ejcts/ezv356

6. Fojtik JP, Costantino TG, Dean AJ: The diagnosis of aortic dissection by emergency medicine ultrasound . J Emerg Med. 2007, 32:191-196. 10.1016/j.jemermed.2006.07.020

7. Kaban J, Raio C: Emergency department diagnosis of aortic dissection by bedside transabdominal ultrasound. Acad Emerg Med. 2009, 16:809-810. 10.1111/j.1553-2712.2009.00448.x

8. Perkins AM, Liteplo A, Noble VE: Ultrasound diagnosis of type a aortic dissection . J Emerg Med. 2010, 38:490-493. 10.1016/j.jemermed.2008.05.013

9. Chenkin J: Diagnosis of aortic dissection presenting as ST-elevation myocardial infarction using point-ofcare ultrasound. J Emerg Med. 2017, 53:880-884. 10.1016/j.jemermed.2017.08.012

10. Rosenberg H, Al-Rajhi K: ED ultrasound diagnosis of a type B aortic dissection using the suprasternal view . Am J Emerg Med. 2012, 30:2081-2085. 10.1016/j.ajem.2011.11.012 